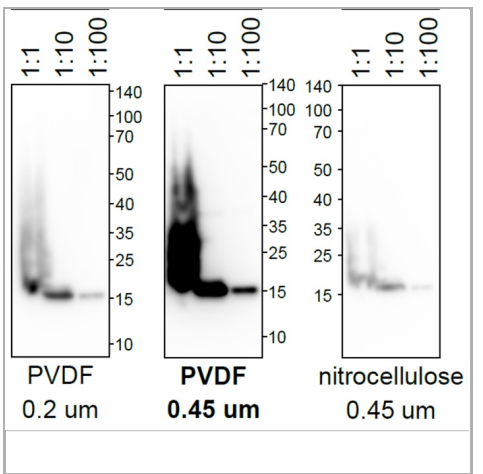

JUN 04, 2020

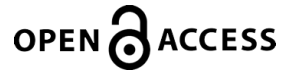

DOI:

dx.doi.org/10.17504/protocol s.io. bg6 kjzcw

Protocol Citation: Gabriele Kaminski Schierle 2020.

Selecting the correct transfer membrane for Western blot enhances detection of alphasynuclein and tau proteins. protocols.io

https://dx.doi.org/10.17504/p rotocols.io. bg6 $6 \mathrm{kzcw}$

License: This is an open access protocol distributed under the terms of the Creative Commons Attribution License, which permits unrestricted use, distribution, and reproduction in any medium, provided the original author and source are credited

Protocol status: Working We use this protocol and it's working

Created: Jun 04, 2020

Last Modified: Jun 04, 2020

PROTOCOL integer ID: 37804

Keywords: Western blot, gel transfer, alpha-synuclein, tau, PVDF, nitrocellulose, fixation

\section{(3) Selecting the correct transfer membrane for Western blot enhances detection of alpha-synuclein and tau proteins}

5 In 1 collection

\author{
Gabriele Kaminski Schierle ${ }^{1}$ \\ ${ }^{1}$ University of Cambridge \\ Molecular Neuroscience Group \\ Tech. support email: gsk20@cam.ac.uk
}

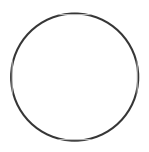

Gabriele Kaminski Schierle

University of Cambridge

\section{ABSTRACT}

The ability to detect proteins by Western blot, particularly from tissue samples, can be hampered by the choice of membrane and whether a fixation step is used. Here we compare three transfer membranes, polyvinylidene difluoride (PVDF) $0.2 \mu \mathrm{m}$, PVDF $0.45 \mu \mathrm{m}$ and nitrocellulose $0.45 \mu \mathrm{m}$ for the detection of rat $\alpha$-synuclein from tissue and recombinant human tau. For $\alpha$-synuclein fixation of the membrane directly after transfer was imperative for detection of the protein and use of the PVDF $0.45 \mu \mathrm{m}$ membrane gave the highest detection signal. For tau, the signal was highest on nitrocellulose $0.45 \mu \mathrm{m}$ and fixation did not enhance the signal. 
MATERIALS

STEP MATERIALS

88 NuPAGE $^{\mathrm{TM}}$ LDS Sample Buffer (4X) Thermo Fisher Catalog \#NP0008

8 NuPAGE $^{\text {TM }} 4-12 \%$ Bis-Tris Protein Gels, $1.5 \mathrm{~mm}$, 15-well Thermo Fisher Catalog \#NP0336PK2

88 NuPAGE ${ }^{\mathrm{TM}}$ MES SDS Running Buffer (20X) Thermo Fisher Catalog \#NP0002

89 Immobilon-PSQ PVDF Membrane 0.2um roll Merck MilliporeSigma (SigmaAldrich) Catalog \#ISEQ00005

Immobilon-P PVDF Membrane, 0.45um, roll Merck MilliporeSigma (SigmaAldrich) Catalog \#IPVH00010

88 Nitrocellulose Transfer Membrane Amersham plc Catalog \#10600002

88 Methanol Contributed by users

88 NuPAGE $^{\mathrm{TM}}$ transfer buffer Thermo Fisher Scientific Catalog \#NP0006

88 PBS Contributed by users

88 Tween-20 Merck MilliporeSigma (Sigma-Aldrich) Catalog \#P9416

8 Rabbit IgG horse radish peroxidase (HRP) linked Whole Ab Contributed by users Catalog \#GENA934-1ML

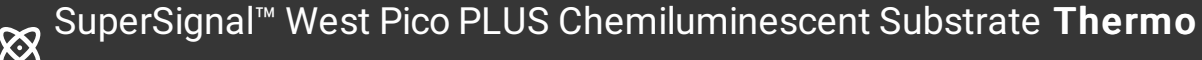
Fisher Catalog \#34579

88 Polyclonal Rabbit Anti-Human Tau Unconjugated Ig fraction Agilent Technologies Catalog \#A002401-2

$88{ }^{\alpha-S y n u c l e i n ~(D 37 A 6) ~ X P ß ~ R a b b i t ~ m A b ~ C e l l ~ S i g n a l i n g ~ T e c h n o l o g y ~ C a t a l o g ~}$ \#4179

89 Rabbit IgG horse radish peroxidase (HRP) linked Whole Ab Contributed by users Catalog \#GENA934-1ML

\section{SAFETY WARNINGS}

(4) Follow safety guidelines and wear correct PPE when handling fication solution containing $4 \%$ paraformalsehyde and $0.1 \%$ gluteraldehyde

1 Protein samples were boiled for 00:03:00 in 1x

\section{NuPAGE $^{\text {TM }}$ LDS Sample Buffer (4X) Thermo Fisher Catalog \#NP0008} and ran on

88 NuPAGE $^{\text {TM }}$ 4-12\% Bis-Tris Protein Gels, 1.5 mm, 15-well Thermo Fisher Catalog \#NP0336PK2

in

8 NuPAGE $^{\text {TM }}$ MES SDS Running Buffer (20X) Thermo Fisher Catalog \#NP0002 using the 


\section{Equipment}

XCell SureLock Mini-Cell Electrophoresis System

Electrophoresis System

Invitrogen

El0001

at $200 \mathrm{~V}$ for $00: 30: 00$

2 Three membranes were selected to test Western blot transfer and detection, PVDF $0.2 \mu \mathrm{m}$

Immobilon-PSQ PVDF Membrane 0.2um roll Merck MilliporeSigma (SigmaAldrich) Catalog \#ISEQ00005

PVDF $0.45 \mu \mathrm{m}$

Q Immobilon-P PVDF Membrane, 0.45um, roll Merck MilliporeSigma (SigmaAldrich) Catalog \#IPVH00010

and nitrocellulose $0.45 \mu \mathrm{m}$

88 Nitrocellulose Transfer Membrane Amersham plc Catalog \#10600002

3 PVDF membranes were first activated with methanol by incubating for 00:03:00 Nitrocellulose membranes do not need to be activated first.

4 The gel was transferred onto each membrane using the

\section{Equipment}

Invitrogen XCell II ${ }^{\mathrm{TM}}$ Blot Module

Western blot transfer module

Invitrogen

El9051

with 


\section{NUPAGE $^{\text {TM }}$ transfer buffer Thermo Fisher Scientific Catalog \#NP0006}

$+20 \%$

\section{Methanol Contributed by users}

at $30 \mathrm{~V}$ for 01:30:00 . The blot module was kept $8^{\circ}$ On ice and surrounded by ice to keep cool and prevent protein damage.

\section{Safety information}

PFA and gluteraldehyde are toxic and should be handled in a chemical hood with appropriate PPE. The fixation solution should never be disposed of down the sink, but disposed of in a serparate container for disposal through correct waste systems.

For detecting $\alpha$-synuclein in tissue samples the membranes were fixed immediately after transfer with 4\% paraformaldehyde (PFA), 0.1\% glutaraldehyde in PBS for 00:30:00. Lack of fixation leads to poor/no $\alpha$-synuclein detection and fixation with only $4 \%$ PFA also leads to lower detection than with the addition of $0.1 \%$ glutaraldehyde.

6 Following disposal of the fixation solution into properly designated containers for removal, the membranes were blocked for $01: 00: 00$ in 5\% BSA in

\section{PBS Contributed by users}

$+0.05 \%$

\section{Tween-20 Merck MilliporeSigma (Sigma-Aldrich) Catalog \#P9416}

\section{(PBS-T) at $8^{\circ}$ Room temperature}

7 The membranes were then incubated either overnight or for $\circlearrowleft$ 01:00:00 at

$8^{\circ}$ Room temperature in primary antibody probing for rat $\alpha$-synuclein 1:1000 dilution

$88 \alpha$-Synuclein (D37A6) XP® Rabbit mAb Cell Signaling Technology Catalog \#4179

8 The membranes were washed three times for $\circlearrowleft 00: 05: 00$ in PBS-T.

9 The membranes were incubated for $01: 00: 00$ at $8^{\circ}$ Room temperature in secondary antibody 1:4000 
10 The membranes were washed five times for 00:05:00 in PBS-T.

11 The membranes were developed in

88 SuperSignal $^{\text {Tm }}$ West Pico PLUS Chemiluminescent Substrate Thermo Fisher Catalog \#34579

and imaged in a

\section{Equipment}

G:Box Chemi

Imaging system

Syngene

XX6 


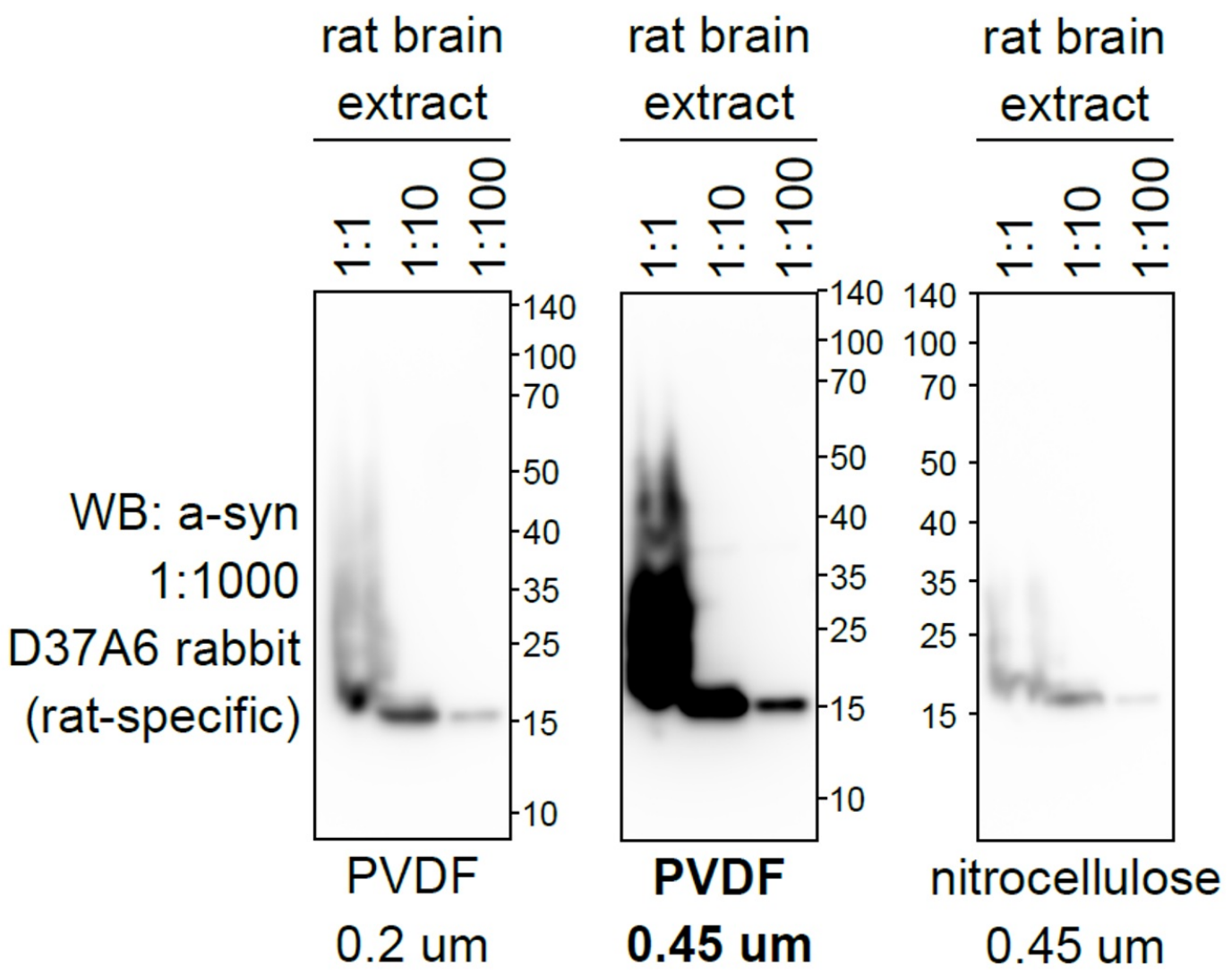

The best signal for detection of alpha-synuclein from rat tissue was obtained on PVDF 0.45 $\mu \mathrm{m}$ with fixation in $4 \%$ PFA and $0.1 \%$ glutaraldehyde.

13 For recombinant human tau, fixation was not required and the membranes were instead immediately blocked for 01:00:00 in 5\% BSA in PBS-T at 8 Room temperature

14 The membranes were then incubated either overnight or for $\circlearrowleft$ 01:00:00 at $8^{\circ}$ Room temperature in primary antibody probing for human tau 1:200 dilution 88 Polyclonal Rabbit Anti-Human Tau Unconjugated Ig fraction Agilent Technologies Catalog \#A002401-2 
15 The membranes were washed three times for 00:05:00 in PBS-T.

16 The membranes were incubated for $\circlearrowleft$ 01:00:00 at $8^{\circ}$ Room temperature in secondary antibody, 1:4000 dilution

88 Rabbit IgG horse radish peroxidase (HRP) linked Whole Ab Contributed by users Catalog \#GENA934-1ML

17 The membranes were washed five times for 00:05:00 in PBS-T.

18 The membranes were developed in Supersignal West Pico (\#34580, ThermoFisher) and imaged in a

\section{Equipment}

G:Box Chemi

NAME

Imaging system

TYPE

Syngene

BRAND

XX6

SKU

\section{9}




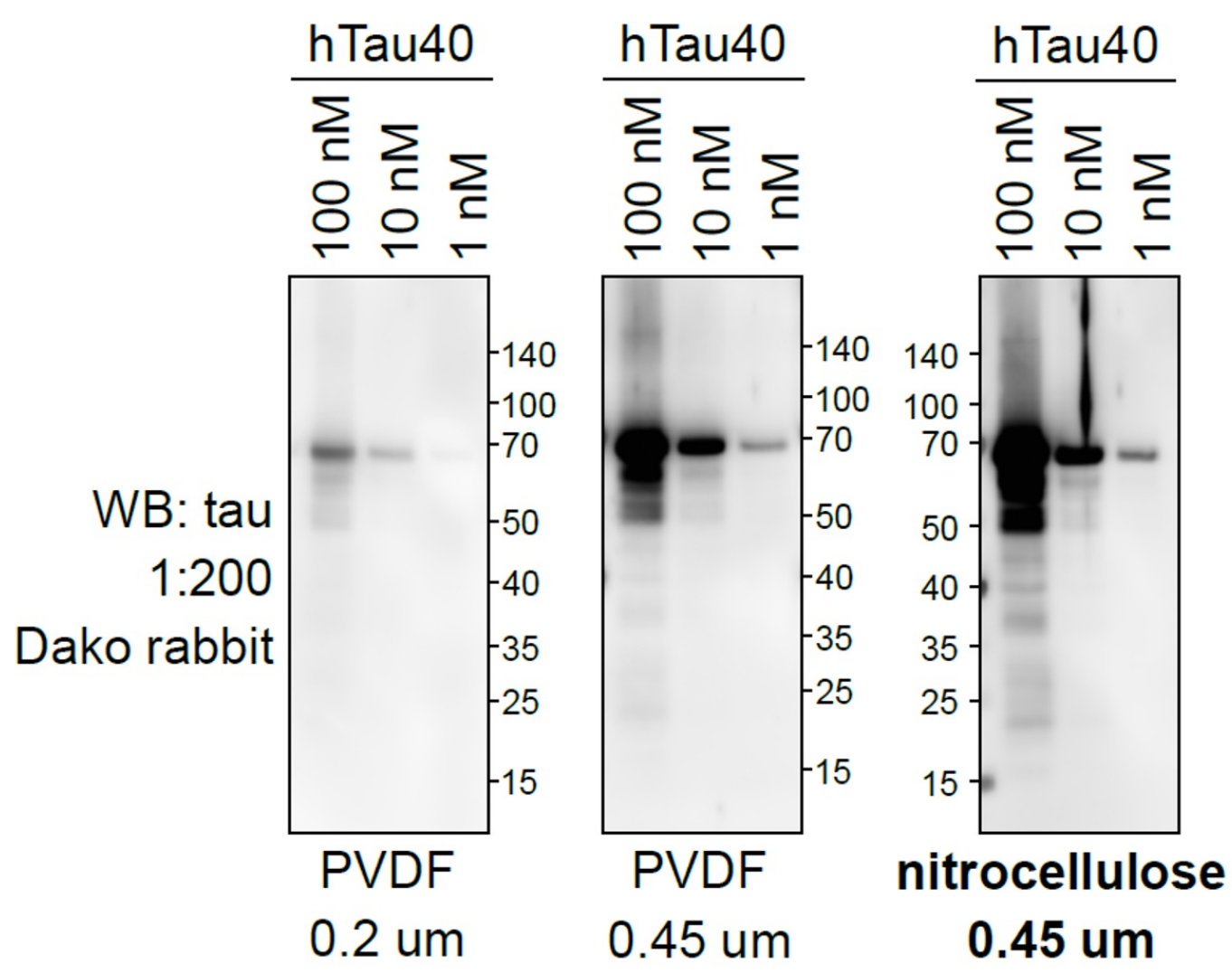

The best signal for recombinant Tau was obtained on nitrocellulose $0.45 \mu \mathrm{m}$.

20 This optimisation was originally performed by Dr Colin Hockings, a former postdoc in the Molecular Neuroscience Group. 